\title{
ÇANÁLISE DA APLICABILIDADE DO FILTRO DIGITAL DE GOERTZEL NA MEDIÇÃO DE DESEQUILÍBRIOS DE TENSÃO
}

\author{
A. R. LIMA \\ Instituto Federal do Rio Grande do Norte \\ alfredo-rodrigues@hotmail.com
}

Submetido 17/06/2017 - Aceito 10/08/2017

DOI: $10.15628 /$ holos.2017.6028

\section{RESUMO}

Este artigo apresenta a utilização de um algoritmo simples de detecção de frequências no cálculo do Fator de Desequilíbrio de Tensão. O algoritmo de Goertzel permite que sejam encontradas, em um sinal distorcido, a amplitude e a fase de uma harmônica específica, sem requerer grande capacidade de armazenamento ou de processamento. Dessa forma, é possível implementar o método das componentes simétricas para o cálculo do fator de desequilíbrio de tensão, utilizando o algoritmo de Goertzel para obter o ângulo de fase e a amplitude da componente fundamental das tensões. A simulação é feita através da ferramenta Scilab. São gerados sinais que correspondem às tensões desequilibradas, tanto por módulo como por ângulo. O programa principal é implementado de forma a fornecer o valor do fator de desequilíbrio para diversas situações. Em seguida são plotados gráficos com as formas de onda das tensões e com os respectivos fasores. A análise dos resultados mostra a simplicidade, utilidade e viabilidade do uso do filtro de Goertzel em aplicações relacionadas com a qualidade da energia elétrica, permitindo que, com menor investimento em hardware, seja possível projetar equipamentos capazes de detectar e mensurar desequilíbrios de forma mais confiável e generalista.

PALAVRAS-CHAVE: Qualidade de energia, Desequilíbrio de tensão, Algoritmo de Goertzel, Instalações elétricas.

\section{ANALYSIS OF APPLICABILITY OF GOERTZEL DIGITAL FILTER IN MEASUREMENT OF VOLTAGE UNBALANCES}

\section{ABSTRACT}

This paper presents the use of a simple frequency detection algorithm in the calculation the Voltage Unbalance Factor. The Goertzel's algorithm allows the amplitude and phase of a specific harmonic can be found in a distorted signal without requiring large storage or processing capacity. Thus, it is possible to implement the symmetric components method to calculate the voltage unbalance factor using the Goertzel algorithm to obtain the phase angle and amplitude of the fundamental component of the voltages. The simulation is done through the Scilab tool. Signals are generated that correspond to the unbalanced voltages, both by module and by angle. The main program is implemented in order to provide the value of the unbalance factor for several situations. Then graphs are plotted with the waveforms of the voltages and their phasors. The analysis of the results shows the simplicity, usefulness and feasibility of the use of the Goertzel filter in applications related to the quality of electric energy, allowing, with less investment in hardware, it is possible to design equipment capable of detecting and measuring imbalances in a more reliable way And generalist.

KEYWORDS: Power Quality, Voltage Imbalance, Goertzel Algorithm, Electrical Installations. 


\section{INTRODUÇÃO}

A medição de desequilíbrios de tensão é de suma importância para as instalações elétricas. Interromper o fornecimento de energia para um circuito no qual foi detectado desvio de módulo ou de fase da tensão de qualquer uma das fases pode evitar danos e até a perda de máquinas elétricas. Entretanto, na grande maioria dos casos, dispositivos de proteção contra desequilíbrio somente são instalados em motores trifásicos, por serem mais caros, justificando o investimento.

A ANEEL (2017), por meio do capítulo 8 do PRODIST, define o desequilíbrio de tensão como a razão entre o módulo da componente de sequência negativa e o módulo da componente de sequência positiva, conforme a Equação (1).

$F D=\frac{\left|V_{2}\right|}{\left|V_{1}\right|} \times 100 \%$

Onde,

- $V_{2}$ - Componente de sequência negativa

- $V_{1}$ - Componente de sequência positiva

Alternativamente, é permitido o uso do método CIGRÉ, que produz resultados bastante próximos do método das componentes simétricas, sendo $2 \%$ o valor limite para o desequilíbrio de tensão estabelecido pela norma (Andrade, Franco, \& Santana, 2015).

Como nem todos os dispositivos dispões de hardware adequado para computar o ângulos de fase das tensões, há a necessidade de se utilizar métodos tais como o CIGRÉ e o NEMA, fornecendo resultados confiáveis, respeitando certas condições (Nunes, 2015).

Ao se desenvolver equipamentos que operem segundo métodos baseados nos valores das tensões de linha, consegue-se proteger motores ou transformadores ligados em triângulo, tendo em vista que tal expressão não envolve os ângulos de fase das tensões, o que permite a construção de equipamentos acessíveis para a maioria dos usuários.

No entanto, quando ocorre a flutuação do neutro, as tensões de linha permanecem equilibradas, mas as tensões de fase se tornam desequilibradas. Vale ressaltar que a fase que possui maior impedância é justamente aquela que é submetida à maior tensão. Esse tipo de problema não é detectado pelos relés de desequilíbrio convencionais, o que poderá causar danos e até a perda de equipamentos monofásicos ou ainda equipamentos trifásicos ligados em estrela.

Outro problema a ser mencionado é a presença de harmônicos. A Equação (1), assim como outras equações utilizadas para o cálculo de desequilíbrio, foram definidas para sistemas que operam na frequência fundamental. Em sistemas com harmônicas, dependendo da forma como o circuito de medição realiza o cálculo do valor eficaz, os valores das tensões podem apresentar erros e, consequentemente, o desequilíbrio pode ser erroneamente estimado. 
A proposta deste trabalho é utilizar do Algoritmo de Goertzel para filtrar a componente fundamental de em um sinal distorcido, encontrando o módulo e a fase dessa componente. Em seguida, utilizar o método das componentes simétricas para calcular o fator de desequilíbrio de tensão. Como será discutido posteriormente, pelas características do algoritmo, não será necessária grande capacidade de armazenamento ou processamento, por parte do hardware, o que permitirá a construção de equipamentos com medições mais confiáveis, sem, no entanto, aumentar o custo de produção.

Inicialmente, é feita uma breve revisão bibliográfica. Posteriormente, no tópico 3, serão apresentadas as ferramentas, os métodos e os materiais utilizados na pesquisa. No tópico 4 são apresentados os resultados por meio de gráficos e tabelas. Finalmente, no tópico 5 , serão feitas as considerações finais.

\section{REVISÃO BIBLIOGRÁFICA}

\subsection{Desequilíbrio de Tensão}

Desequilíbrio de tensão é uma condição na qual pelo menos uma das fases possui o valor do módulo diferente, ou ainda, a defasagem angular entre pelo menos um par de fases é diferente de 120 graus.

São causados por cargas monofásicas diversas inseridas na rede de distribuição, fazendo que a corrente nos circuitos de distribuição sejam desequilibradas. As correntes desequilibradas, ao passarem por linhas de impedância não nula, provocam diferentes quedas de tensão. Tais quedas de tensão acarretam em diferentes valores de tensão nas três fases (ARÃO, 2014).

Os desequilíbrios de tensão trazem diversas consequências tanto para cargas trifásicas como monofásicas. No caso de motores trifásicos, por exemplo, o desequilíbrio está associado ao aparecimento de componente de sequência negativa, a qual é responsável por causar os seguintes problemas (REZENDE \& SAMESIMA, 2012):

- Aumento do valor eficaz da corrente nos enrolamentos;

- Elevação da temperatura de operação dos condutores dos enrolamentos;

- Redução do conjugado, devido à existência de torque contrário ao sentido normal de rotação;

Aparecimento de vibrações mecânicas indesejadas;

- Aumento das perdas;

- Diminuição da vida útil.

\subsection{Cálculo do Desequilíbrio de Tensão}

A ANEEL, através do módulo 8 do PRODIST, estabelece a forma de cálculo do fator de desequilíbrio baseando-se na teoria das componentes simétricas. As componentes de sequência 
positiva e negativa podem ser encontradas através das equações (2) e (3), respectivamente (COTRIM, 2009):

$$
\begin{aligned}
& V_{1}=\frac{1}{3}\left(V_{A}+a V_{B}+a^{2} V_{C}\right) \\
& V_{2}=\frac{1}{3}\left(V_{A}+a^{2} V_{B}+a V_{C}\right)
\end{aligned}
$$

\subsection{Métodos Alternativos Para o Cálculo do Desequilíbrio de Tensão}

A aplicação do método das componentes simétricas para o cálculo do desequilíbrio traz um grande inconveniente para vários dispositivos de medição. Grande parte dos equipamentos destinados a esse fim não possui hardware apropriado para medir o ângulo de fase das tensões. Por isso, alguns métodos alternativos são comumente utilizados, tanto nos trabalhos científicos como no contexto industrial, a fim de quantificar o fator de desequilíbrio baseando-se nos valores das tensões de linha (Nunes, 2015). Tais métodos são discutidos nas próximas subseções.

\subsubsection{Método NEMA}

O método NEMA considera a média aritmética das tensões de linha e o máximo desvio entre as tensões de linha e o valor dessa média. É calculado conforme a Equação (4).

$$
F D \%=100 \times \frac{\Delta V}{V_{M E D}}
$$

Onde,

- $\Delta V$ - Máxima variação de tensão em relação ao valor médio das tensões de linha;

- $V_{M E D}$ - Média aritmética das tensões de linha.

A Equação (5) é de fácil aplicação, tanto a nível de hardware como de software, pois exige apenas que se saiba os valores eficazes das tensões. Também é necessária a implementação de um algoritmo para se definir qual é o máximo valor de tensão entre as três fases e o valor da média aritmética correspondente a essas tensões.

\subsubsection{Método CIGRÉ}

O método CIGRÉ calcula o desequilíbrio através de uma grandeza adimensional que depende do valor das tensões de linha. O fator de desequilíbrio é dado pela Equação (5).

$$
F D \%=100 \times \sqrt{\frac{1-\sqrt{3-6 \beta}}{1+\sqrt{3-6 \beta}}}
$$

Onde, 


$$
\beta=\frac{\left|V_{A B}\right|^{4}+\left|V_{B C}\right|^{4}+\left|V_{C A}\right|^{4}}{\left.\left|V_{A B}\right|^{2}+\left|V_{B C}\right|^{2}+\left|V_{C A}\right|^{2}\right)^{2}}
$$

Se, por um lado, o referido método não necessita de definição de valor máximo ou de média aritmética, possui operações matemáticas um pouco mais complexas que o método NEMA.

\subsubsection{Método IEEE}

Outro método conhecido para cálculo do fator de desequilíbrio foi adotado pelo IEEE. Expressa a razão entre a maior diferença das tensões de linha e o somatório dessas tensões. É dado pela Equação (7).

$$
F D \%=\frac{3\left(V_{M A X}-V_{M I N}\right)}{V_{A B}+V_{B C}+V_{C A}} \times 100
$$

\subsection{Desvantagem dos Métodos Alternativos}

Segundo Oliveira, Filho e Pinto (2006), no caso de desvio nas magnitudes das tensões, os métodos alternativos de cálculo do desequilíbrio não apresentam erros significativos quando comparados ao método das componentes simétricas, com exceção do método IEEE, que apresenta erros consideráveis, fornecendo valores mais altos de desequilíbrio. No entanto, caso o desequilíbrio seja causado por desvio nos valores dos ângulos de fase, o método NEMA também pode apresentar erros representativos, dependendo do nível de desequilíbrio.

O método CIGRÉ foi o único que apresentou resultados praticamente iguais aos do método das componentes simétricas. Entretanto, diante da possibilidade de ocorrer um deslocamento no neutro, situação na qual as tensões de linha permanecem inalteradas enquanto as tensões de fase apresentam valores distintos entre si, o método CIGRÉ fornecerá valores nulos de desequilíbrio.

Nessas condições, enquanto uma ou duas fases podem apresentar subtensão, uma ou duas fases podem apresentar sobretensão. Os equipamentos de proteção contra desequilíbrio baseados na medição de tensões de linha não podem detectar esse problema, ficando as cargas monofásicas desprotegidas contra os efeitos desse distúrbio, principalmente as cargas de maior impedância, que ficam submetidas às sobretensões.

Também é necessário avaliar como o desequilíbrio é afetado pela presença de componentes harmônicas. Os equipamentos de medição normalmente calculam o valor eficaz da tensão nas três fases. Se a tensão em alguma das fases possui conteúdo harmônico, é natural que o valor do fator de desequilíbrio seja alterado. Logo, independente do método alternativo escolhido, é necessário dispor de filtros para impedir que as harmônicas interfiram no resultado do cálculo de desequilíbrio.

Com o algoritmo de Goertzel, é possível realizar a filtragem dos harmônicos e encontrar a amplitude da componente de $60 \mathrm{~Hz}$ nas três fases, o que permite a aplicação dos métodos 
alternativos para o cálculo do fator de desequilíbrio. Por outro lado, tal algoritmo também consegue detectar o ângulo de fase das tensões, permitindo, de maneira simples, a aplicação do método das componentes simétricas.

\subsection{O Algoritmo de Goertzel}

Utilizado geralmente em sistemas DTMF, o algoritmo de Goertzel consiste num filtro digital do tipo IIR. Derivado da DFT, é utilizado em situações nas quais existe a necessidade de identificar harmônicos presentes em sinais (CHASSAING \& REAY, 2008). Entretanto, quando comparado com a DFT e até mesmo com a FFT, apresenta maior simplicidade de implementação e requer menor capacidade computacional, tornando-se uma excelente alternativa quando se deseja produzir um hardware dispondo de poucos recursos. O algoritmo de Goertzel é indicado quando se deseja analisar poucas componentes de frequência, do contrário, o referido algoritmo já não traz a mesma eficiência que a FFT (ARAÚJO, LIRA, \& FERREIRA, 2012).

\subsubsection{Implementação do algoritmo de Goertzel}

O primeiro passo da implementação é a definição da frequência de amostragem e do tamanho do vetor de amostras. Para cada frequência analisada, existe um valor $k$, que é dado pela Equação (5).

$$
k=N \frac{f_{B}}{f_{A}}
$$

Onde:

- $f_{B}$ - Frequência buscada;

- $\quad N$ - Tamanho do vetor de amostras

- $f_{A}$ - Frequência de amostragem

A combinação conveniente da quantidade de amostras e da frequência de amostragem permite que os valores de $\mathrm{k}$ sejam números inteiros que representem a ordem da harmônica analisada.

Depois de definidos os parâmetros iniciais, são utilizadas as equações (10) e (11), (OSORIO \& VARGAS, 2010).

$$
\begin{aligned}
& S_{k}(n)=x(n)+2 \cos \left(\frac{2 k \pi}{N}\right) S_{k}(n-1)-S_{k}(n-2) \\
& Y_{k}(n)=S_{k}(n)-e^{-j\left(\frac{2 k \pi}{N}\right)} S_{k}(n-1)
\end{aligned}
$$

Onde:

- $\quad x(n)$ - enésima amostra da tensão;

- $\quad S_{k}(n)$ - Saída atual; 
- $S_{k}(n-1)$ - Saída anterior;

- $S_{k}(n-2)$ - Saída ante anterior;

- $Y_{k}(n)$ - Resposta final do algoritmo.

As equações (6) e (7) têm a forma de um filtro digital recursivo com pequeno número de operações matemáticas, motivo pelo qual podem ser aplicadas em sistemas que dispões de recursos escassos de hardware (LIRA, FREIRE, COSTA, MACEDO, \& LUCIANO, 2005).

A Equação (6) é aplicada de forma iterativa, com $n$ variando desde a unidade até a quantidade de amostras do vetor. Após a última iteração aplica-se a Equação (7), resultando em um número complexo na forma exponencial. Uma vez que esse número tem parte real e imaginária, é possível realizar manipulações matemáticas para encontrar os valores do seu módulo e do seu ângulo. Os valores encontrados dessas duas grandezas correspondem, respectivamente, à amplitude da onda senoidal e o seu deslocamento angular em relação ao eixo das ordenadas, considerando um gráfico no domínio do tempo.

\section{MATERIAIS E MÉTODOS}

\subsection{Definição dos Parâmetros Iniciais}

\subsubsection{Frequência de Amostragem}

A definição da frequência de amostragem deve seguir o critério de Nyquist, correspondendo ao dobro da maior frequência contida no sinal a ser amostrado (CARVALHO, 2009). A ANNEL estabelece que, no contexto das harmônicas, os equipamentos de medição devem ser capazes de detectar as componentes de até 25a ordem. Nessas condições, para garantir que não ocorra aliasing, seria necessária uma taxa de amostragem de $3 \mathrm{kHz}$. Entretanto, foi adotada uma frequência de $12 \mathrm{kHz}$, com o objetivo de garantir maior resolução nos resultados das medições.

\subsubsection{Comprimento do Vetor de Amostras}

A definição do comprimento do vetor das amostras influencia em dois detalhes de grande importância: A resolução no valor das medições e a capacidade de armazenamento do hardware. Vetores com mais elementos permitem que a resposta tenha maiores níveis de resolução. Porém, exige mais espaço de memória RAM, por parte do hardware. Uma vantagem de se utilizar o algoritmo de Goertzel reside no fato de que o tamanho desse vetor não precisa ser uma potência de 2 .

\subsection{Implementação}

A metodologia consiste na utilização da ferramenta Scilab para geração de formas de onda correspondentes a tensões trifásicas distorcidas e desequilibradas. Tais tensões podem 
conter componentes harmônicas até a 25a ordem, sendo a amplitude dessas componentes ajustadas no próprio código, antes de cada simulação. É possível modificar tanto os módulos como os ângulos de fase de cada tensão, a fim de impor diferentes níveis de desequilíbrio. Para efeito de análise, são consideradas todas as possibilidades de desequilíbrios: por módulo, por ângulo e por módulo e ângulo.

Em seguida as amostras das tensões trifásicas são utilizadas como variáveis de entrada do algoritmo de Goertzel, que faz a filtragem, analisando apenas a componente de $60 \mathrm{~Hz}$. Para cada uma das três fases, o algoritmo fornecerá o módulo e o ângulo do sinal amostrado. Finalmente, com base nos valores dos módulos e dos ângulos calculados, aplica-se a Equação (1), obtendo o fator de desequilíbrio de tensão. Na Figura 1 é mostrado o fluxograma que representa a estrutura do código programado no Scilab.

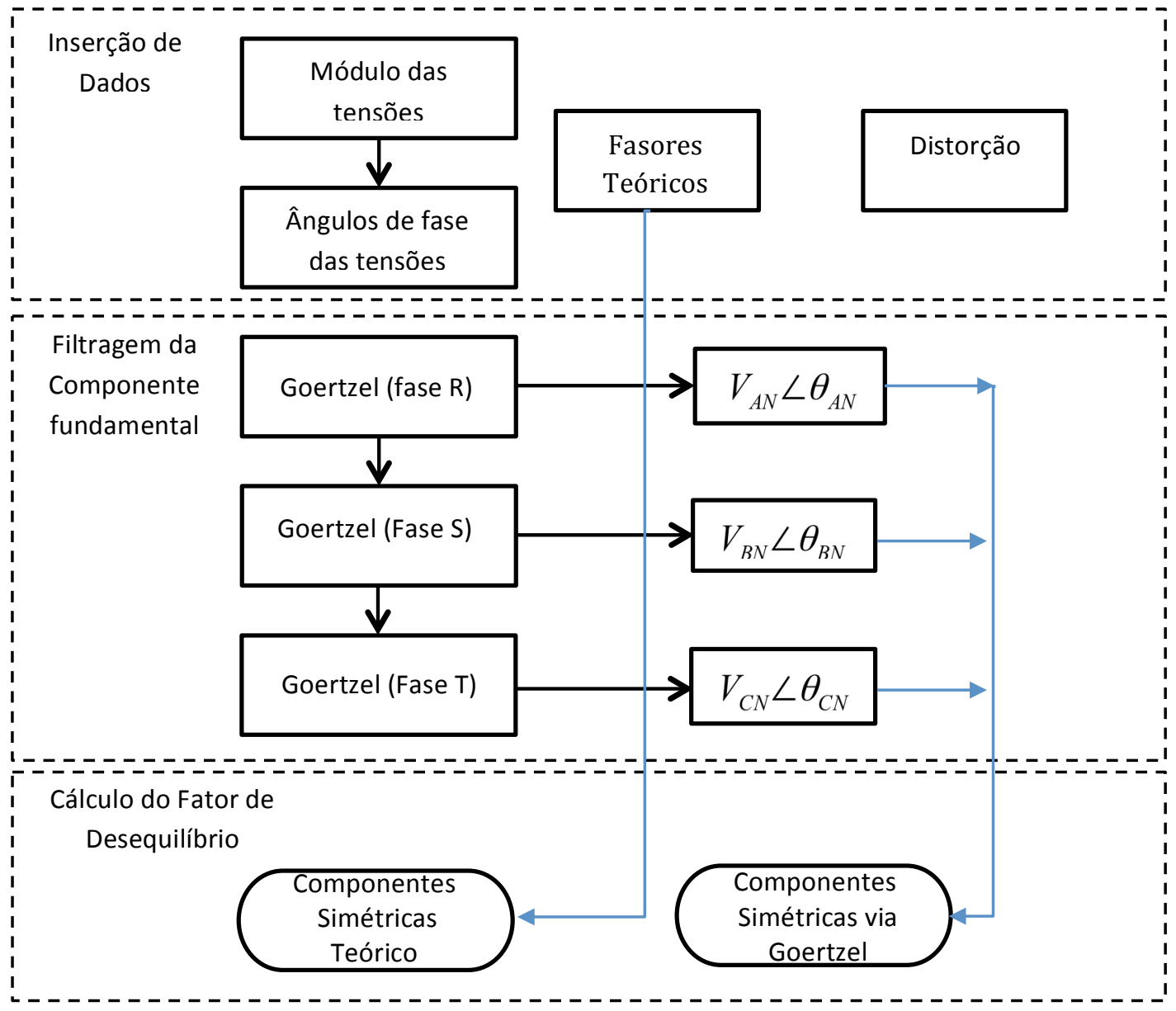

Figura 1: Fluxograma do programa feito em Scilab 


\section{RESULTADOS E DISCUSSÕES}

\subsection{Simulação Sem Harmônicos}

\subsubsection{Magnitudes desequilibradas}

Na primeira simulação os ângulos das tensões nas três fases foram mantidos na condição de normalidade. Foram calculados os valores do fator de desequilíbrio conforme o método das componentes simétricas. A Tabela 1 contém o valor das magnitudes e dos ângulos de fase calculados através do algoritmo de Goertzel, além do valor do fator de desequilíbrio resultante da aplicação do método das componentes simétricas.

Tabela 1: Desequilíbrio causado pela alteração das magnitudes

\begin{tabular}{c|c|c|c|c|c|c}
\hline Fase & $\begin{array}{c}\text { Magnitudes } \\
\text { Impostas }\end{array}$ & $\begin{array}{c}\text { Ângulos } \\
\text { Impostos }\end{array}$ & $\begin{array}{c}\text { Magnitudes } \\
\text { Calculadas }\end{array}$ & $\begin{array}{c}\text { Ângulos } \\
\text { Calculados }\end{array}$ & $\begin{array}{c}\text { FD (Teórico) } \\
\text { (\%) }\end{array}$ & $\begin{array}{c}\text { FD (Experimental) } \\
\text { (\%) }\end{array}$ \\
\hline A & 230,0 & 0 & 230,0 & $-1,8000006$ & & \\
\cline { 1 - 4 } B & 220,0 & -120 & 220,0 & $-121,80009$ & 2,624115 & 2,624115 \\
\cline { 1 - 4 } C & 210,0 & 120 & 210,0 & 118,20009 & & \\
\hline
\end{tabular}

A Figura 2 mostra as tensões nas três fases e o diagrama fasorial. Os fasores tracejados representam as tensões calculadas por meio do algoritmo de Goertzel.

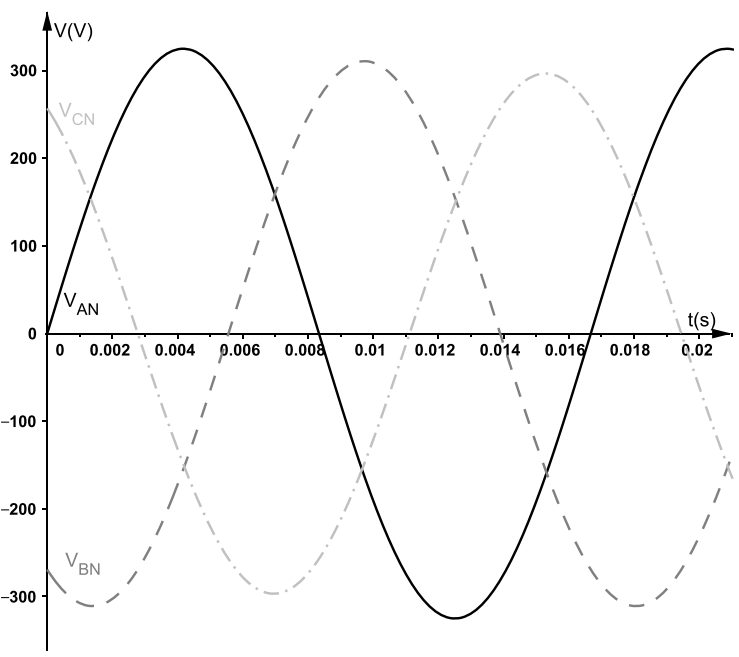

a)

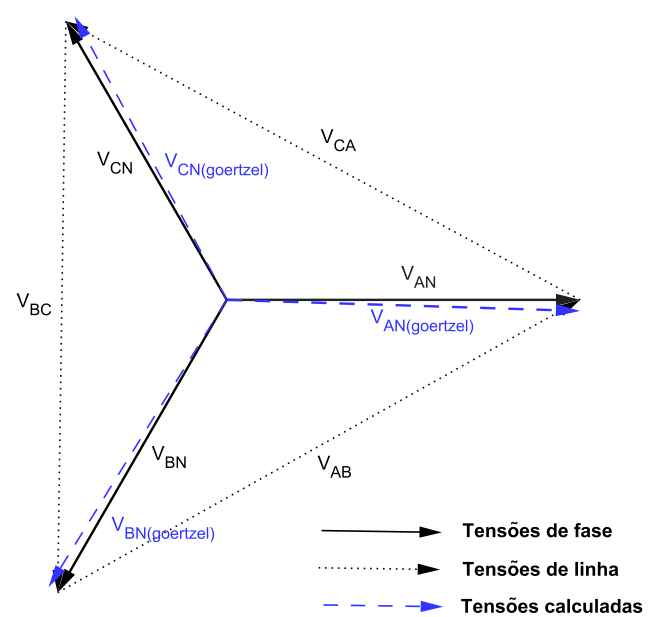

b)

Figura 2: a) Tensões impostas; b) Diagrama fasorial das tensões impostas e calculadas.

Observa-se que os valores das magnitudes foram calculados de forma exata, enquanto os valores dos ângulos apresentaram erros em relação aos ângulos impostos. Entretanto, como o 
erro no cálculo do ângulo é igual nas três fases, o resultado do fator de desequilíbrio não sofreu alteração.

\subsection{2 Ângulos desequilibrados}

Em seguida as magnitudes foram ajustadas em 220 volts, enquanto os ângulos das tensões foram ajustados de forma desequilibrada, conforme indicado na Tabela 2.

Tabela 2: Desequilíbrio causado pela alteração dos ângulos

\begin{tabular}{c|c|c|c|c|c|c}
\hline Fase & $\begin{array}{c}\text { Magnitudes } \\
\text { Impostas }\end{array}$ & $\begin{array}{c}\text { Ângulos } \\
\text { Impostos }\end{array}$ & $\begin{array}{c}\text { Magnitudes } \\
\text { Calculadas }\end{array}$ & $\begin{array}{c}\text { Ângulos } \\
\text { Calculados }\end{array}$ & $\begin{array}{c}\text { FD (Teórico) } \\
\text { (\%) }\end{array}$ & $\begin{array}{c}\text { FD (Experimental) } \\
\text { (\%) }\end{array}$ \\
\hline A & 220,0 & 5 & 220,0 & 3,2000033 & & \multirow{2}{*}{5,1717281} \\
\cline { 1 - 5 } B & 220,0 & -120 & 220,0 & $-121,80009$ & 5,1717281 & \\
\cline { 1 - 4 } C & 220,0 & 115 & 220,0 & 113,20009 & & \\
\hline
\end{tabular}

A Figura 3 mostra as tensões nas três fases e o diagrama fasorial para essa situação.

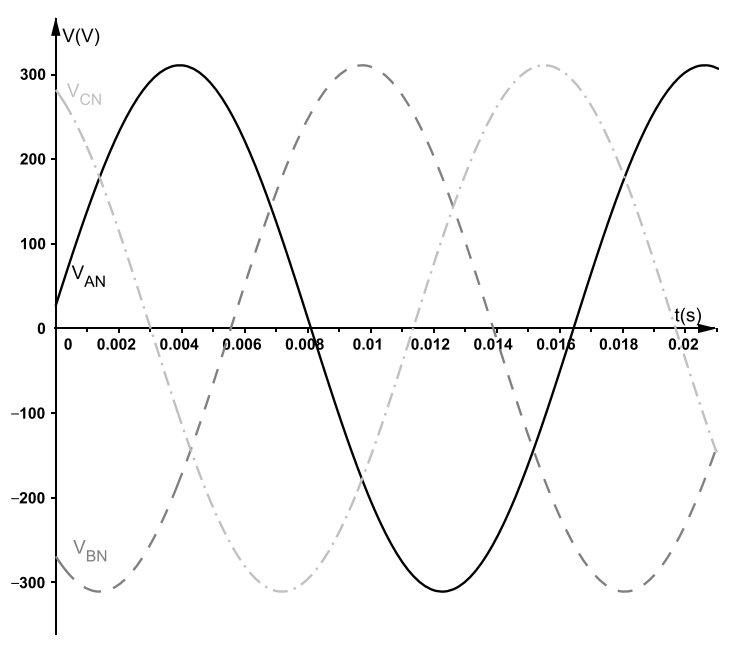

a)

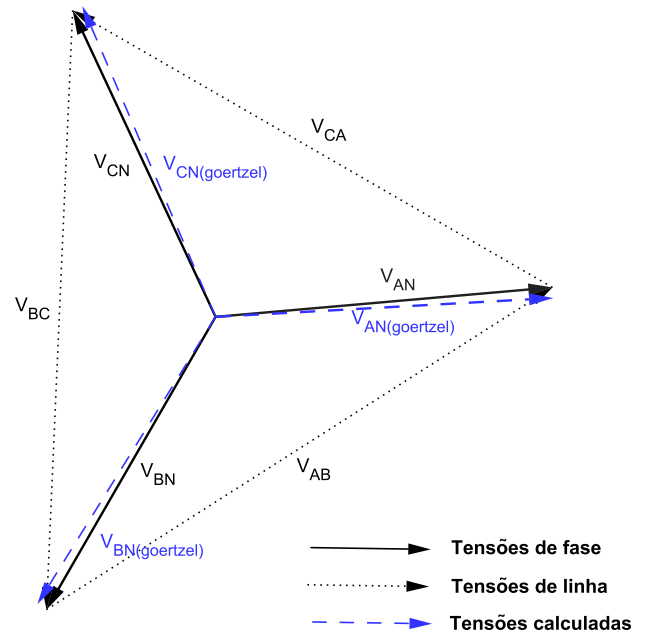

b)

Figura 3: a) Tensões impostas; b) Diagrama fasorial das tensões impostas e calculadas

Nesse caso, observação do diagrama fasorial permite perceber rapidamente a existência de desequilíbrio, o que não seria possível apenas observando as formas de onda em função do tempo.

\subsubsection{Magnitudes e ângulos desequilibrados}

Em seguida, tanto os módulos como as magnitudes das tensões têm seus valores ajustados de forma desequilibrada, conforme mostrado na Tabela 3.

Tabela 3: Desequilíbrio causado pela alteração dos ângulos 


\begin{tabular}{c|c|c|c|c|c|c}
\hline Fase & $\begin{array}{c}\text { Magnitudes } \\
\text { Impostas }\end{array}$ & $\begin{array}{c}\text { Ângulos } \\
\text { Impostos }\end{array}$ & $\begin{array}{c}\text { Magnitudes } \\
\text { Calculadas }\end{array}$ & $\begin{array}{c}\text { Ângulos } \\
\text { Calculados }\end{array}$ & $\begin{array}{c}\text { FD (Teórico) } \\
\text { (\%) }\end{array}$ & $\begin{array}{c}\text { FD (Experimental) } \\
\text { (\%) }\end{array}$ \\
\hline A & 230,0 & 5 & 230,0 & 3,2000033 & & \multirow{2}{*}{5,8588788} \\
\cline { 1 - 5 } B & 220,0 & -120 & 220,0 & $-121,80009$ & 5,8588788 & \\
\cline { 1 - 4 } C & 210,0 & 115 & 210,0 & 113,20009 & & \\
\hline
\end{tabular}

$\mathrm{Na}$ Figura 4 são mostradas as formas de onda das tensões e o respectivo diagrama farosial.

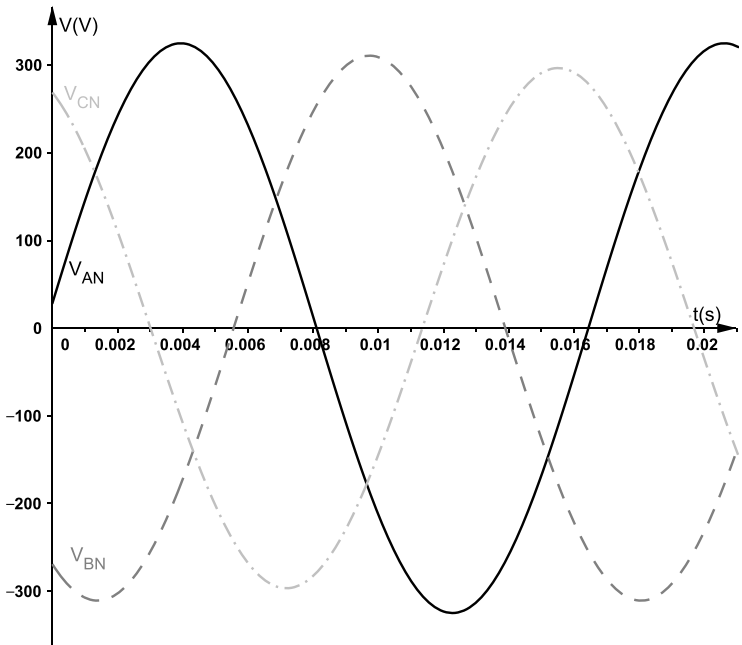

a)

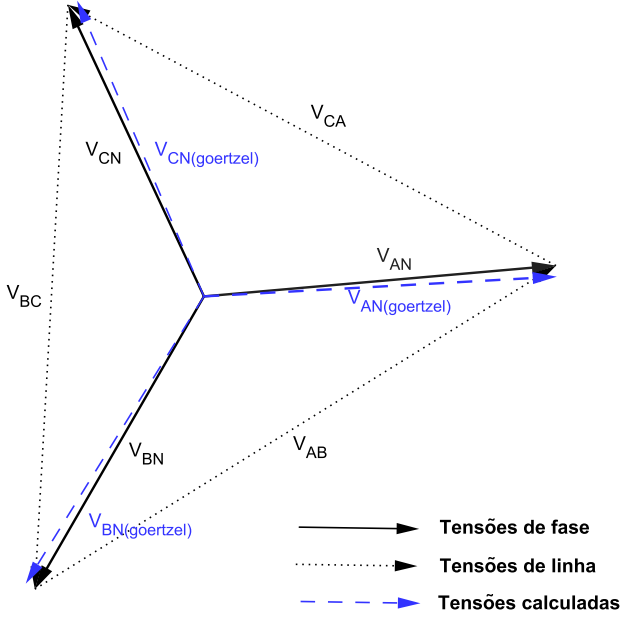

b)

Figura 4: a) Tensões impostas; b) Diagrama fasorial das tensões impostas e calculadas.

A análise conjunta das formas de onda e do diagrama fasorial permite que se tenha boa noção do nível de desequilíbrio existente em determinado circuito. Também é possível distinguir, com maior facilidade, se sua causa é oriunda de desvios de ângulos ou de magnitudes.

O próximo passo é avaliar o quanto os resultados dos cálculos podem ser afetados pela presença de componentes harmônicos, o que será abordado na próxima subseção.

\subsection{Simulação Com Harmônicos}

Até agora, somente foram consideradas ondas puramente senoidais no cálculo do desequilíbrio. É necessário verificar o quanto a presença de harmônicos afetará o cálculo do desequilíbrio. Para isso, a última simulação é repetida, porém com a inserção de componentes harmônicos nas três fases. O resultado é mostrado na Tabela 4.

Tabela 4: Desequilíbrio causado pela alteração dos ângulos e magnitudes na presença de harmônicos

\begin{tabular}{l|l|l|l|l|l|l}
\hline Fase & Magnitudes & Ângulos & Magnitudes & Ângulos & FD (Teórico) & FD (Experimental) \\
\hline
\end{tabular}




\begin{tabular}{c|c|c|c|c|c|c}
\hline & Impostas & Impostos & Calculadas & Calculados & (\%) & (\%) \\
\cline { 1 - 5 } A & 230,0 & 5 & 230,0 & 3,2000033 & & \\
\cline { 1 - 4 } B & 220,0 & -120 & 220,0 & $-121,80009$ & 5,8588788 & 5,8588788 \\
\hline C & 210,0 & 115 & 210,0 & 113,20009 & & \\
\hline
\end{tabular}

Percebe-se que os valores das magnitudes e ângulos calculados, assim como o valor do fator de desequilíbrio, permaneceram iguais aos da simulação anterior. Logo, a filtragem da componente fundamental da tensão nas três fases foi feita eficientemente pelo algoritmo de Goertzel. NA Figura 5 são mostradas as formas de onda e o diagrama fasorial das tensões.

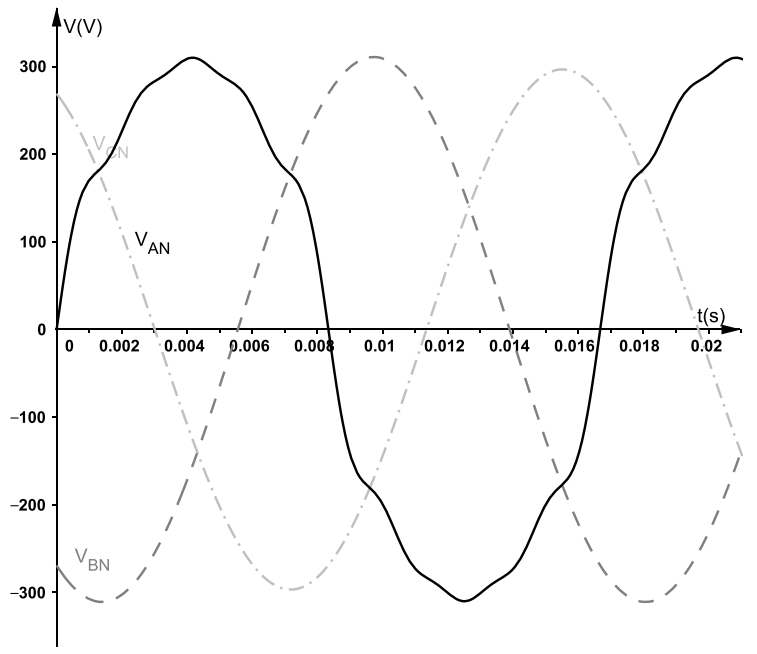

a)

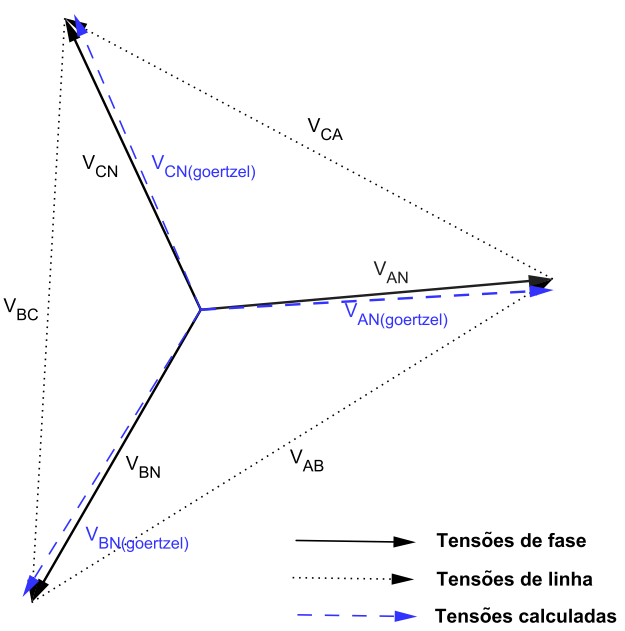

b)

Figura 5: a) Tensões impostas; b) Diagrama fasorial das tensões impostas e calculadas

A Figura 5 permite lembrar o quanto é mais difícil implementar um circuito de estimação de fase quando o sinal apresenta harmônicos. Circuitos baseados em detecção de zero, por exemplo, podem operar de forma errada, já que um sinal com harmônicos pode apresentar várias cristas e vales.

\section{CONSIDERAÇÕES FINAIS}

Os resultados apresentados permitem concluir que, apesar de possuir fácil implementação, o algoritmo de Goertzel oferece resultados confiáveis e satisfatórios para a detecção, cálculo e medição de componentes de frequência presentes em um sinal. Uma vez que não precisa armazenar muitas variáveis durante sua execução, pode ser implementado em sistemas que dispõem de poucos recursos de hardware.

Ao se aplicar um filtro digital de baixo custo em sistemas de medição ou monitoramento de desequilíbrio de tensão, permite-se que consumidores de energia, que até então não tinham 
acesso a essa informação, consigam detectar esse problema, mensurá-lo e, consequentemente, se precaver dos riscos oferecidos.

Um dispositivo de proteção contra desequilíbrios é essencial para a proteção de motores trifásicos. Se ele opera segundo os métodos, NEMA, CIGRÉ ou IEEE, é capaz de proteger máquinas trifásicas, já que se baseia nos valores de tensões de linha. Se opera segundo o método das componentes simétricas, conforme a Equação (1), é capaz de proteger tanto máquinas trifásicas como equipamentos monofásicos ligados em qualquer uma das fases de um quadro de distribuição trifásico.

Atualmente, apenas as máquinas trifásicas são equipadas com dispositivos de proteção contra desequilíbrios. Na grande maioria dos casos, as cargas monofásicas estão expostas às variações nocivas de tensão. Porém, com a possibilidade de implementação com baixo custo de um medidor de desequilíbrio, será possível proteger, desde que acionado um dispositivo de manobra, todas as cargas alimentadas por uma fonte trifásica.

A utilização do algoritmo de Goertzel permitiu que o desequilíbrio de tensão fosse mensurado através de um método mais completo e generalista, detectando desequilíbrios por módulo, por fase e por flutuação do neutro. Além disso, os resultados se mantiveram constantes mesmo com a inserção de harmônicas, o que propicia maior confiabilidade e fidelidade nos resultados das medições.

\subsection{Possíveis Contribuições para a Qualidade de Energia}

Uma vez que esse algoritmo detecta componentes harmônicos presentes em um sinal, pode ser utilizado para detecção e monitoramento de distorção harmônica, apresentando-se como uma alternativa em relação às transformadas de Fourier. Com base na detecção de fase de componentes harmônicas, é possível implementar equipamentos capazes de medir a defasagem angular entre sinais de corrente e tensão, permitindo medir potência ativa, reativa, fator de potência e grandezas associadas.

Desde que implementada a utilização do método das componentes simétricas em um dispositivo de baixo custo, seria possível agregar a funcionalidade de visualização, no próprio equipamento, do diagrama fasorial das tensões. Isso permitiria ações mais rápidas de detecção de problemas e tomadas de decisões. Tudo isso, contribuindo para a melhoria do funcionamento das instalações elétricas e para a diminuição de ocorrências relacionadas a problemas de qualidade de energia.

Portanto, a utilização dessa ferramenta de simples implementação permite que grandes benefícios, em termos de qualidade de energia, sejam alcançados por grande parte dos usuários e unidades consumidoras sem necessitar de alto investimento em hardware.

\section{REFERÊNCIAS}

Andrade, M. V., Franco, J. J., \& Santana, H. D. (2015, Julho). Influência da Expansão das Redes Bifásicas no Desequilíbrio de Tensão - Caso Exemplo - Empresa de Tratamento de Agua. 
Anais: Conferência Brasileira Sobre Qualidade de Energia. Campina Grande, PB, Brasil, 11.

ANEEL - Agência Nacional de Energia Elétrica (2017). Procedimentos de Distribuição de Energia Elétrica no Sistema Elétrico Nacional - PRODIST, 8a Ed, Brasília, DF, Brasil.

ARAÚJO, J. F., LIRA, G. R., \& FERREIRA, T. V. (2012, Maio). Comparação de Técnicas de Estimação de Harmônicos em Sistemas de Potência. Anais: Simpósio Brasileiro de Sistemas Elétricos. Goiânia, GO, Brasil, 4.

ARÃO, L. F. L. (2014). Avaliação Comparativa Entre Métodos Para Atribuição De Responsabilidades Devido Ao Desequilíbrio De Tensão (Dissertação de Mestrado). Universidade Federal de Brasília - UNB, Brasília, DF, Brasil.

CARVAlHO, R. M. (2009). Comunicações Analógicas e Digitais. São Paulo: LTC.

CHASSAING, R., \& REAY, D. (2008). Digital Signal Processing and Aplications with the TMS320C6713 and TMS320C6416 DSK (2a ed.). Hoboken: John Wiley \& Sons..

COTRIM, A. A. (2009). Instalações Elétricas (5a ed.). São Paulo: Pearson.

Lima, J. B., Souza, R. M., Oliveira, H. M., \& Souza, M. M. (2004). Decodificação de Sinais DTMF via Transformada Aritmética de Fourier (Dissertação de Mestrado). Universidade Federal de Pernambuco - UFPE, Recife, PE, Brasil.

LIRA, J. G., FREIRE, R. C., COSTA, E. G., MACEDO, E. C., \& LUCIANO, B. A. (2005, novembro). Sistema de avaliação espectral para sistemas elétricos baseado em microcontrolador. Anais: Ecuentro de Potencia, Instrumentación y Medidas. Montevideu, MO, Uruguai, 6.

Nunes, E. J. (2015, Julho). Redução de Desequilíbrios de Correntes em Alimentadores de Distribuição. Anais: Conferência Brasileira Sobre Qualidade de Energia. Campina Grande, PB, Brasil, 11.

Oliveira, M. A., Filho, A. d., \& Pinto, M. G. (2006, novembro) Análise da Sensibilidade do Desequilíbrio de Tensão Frente Às Variações nos Ângulos e Magnitudes das Tensões. Anais: Congreso Internacional De Distribuición Eléctrica, Buenos Aires, PBA, Argentina, 6.

OSORIO, J. A. C.; VARGAS, J. A. M.; ESCOBAR, J. A. M. (2010). Alternativa al análisis em frecuencia de la FFT mediante el algoritmo Goertzel. Scientia et Technica, 1(44), 217-222.

REZENDE, P. H. O.; SAMESIMA, M. I. (2012). Efeito do Desequilíbrio de Tensões de Suprimento nos Motores de Indução trifásicos. Horizonte Cientîfico, 6(2). 